\title{
1.2. Nuevas herramientas de la comunicación. Un estudio acerca del amor, la amistad, la educación y el trabajo en jóvenes universitarios
}

\section{Belmes, Débora Irina [ver currículum del autor, docente de la Facultad de Diseño y Comunicación]}

Abstract del proyecto:

Esta materia se ubica en el segundo año de las carreras de Publicidad y Relaciones Públicas permitiendo a los estudiantes formarse en el conocimiento y manejo de herramientas metodológicas propias de los ámbitos académicos. En la Licenciatura en Negocios, Diseño y Comunicación la asignatura se encuentra ubicada en el segundo semestre y apunta a afianzar los conocimientos metodológicos adquiridos por los estudiantes en sus carreras de grado, como así los orienta y ejercita en el diseño, proyecto y concreción de un trabajo de investigación. Asimismo forma parte del grupo Ensayos Contemporáneos cuyos objetivos se vinculan a la

\section{Cuadernos del Centro de Estudios de Diseño y Comunicación Nº 63}

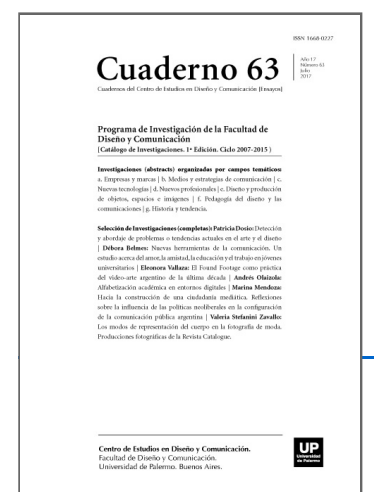

descargar PDF ver índice de la publicación

Ver todos los libros de la publicación

compartir en Facebook
ISSN: 1668-0227

Programa de

Investigación de la

Facultad de Diseño y

Comunicación

Año XVII, Julio 2017, Buenos Aires, Argentina | 352 páginas

realización de documentos escritos de acuerdo a las normas académicas y del desarrollo de las habilidades necesarias para la reflexión sobre temáticas vinculadas al acontecer actual. El presente trabajo está centrado en las denominadas Tecnologías de la información y la comunicación (TIC) usadas por jóvenes universitarios entre dieciocho y veintiocho años. Considerando la institución universitaria como un ámbito adecuado para la observación, el estudio y el análisis de fenómenos contemporáneos, se propone analizar algunos de los modos en que estos dispositivos afectan los procesos de subjetivación, en especial los referidos a la construcción, sostenimiento y finalización de vínculos significativos.

Palabras clave: estudio e investigación - publicidad - relaciones públicas. 
Este trabajo se llevó a cabo en el marco de la materia Metodología de Investigación que se dictó el primer cuatrimestre de 2011 a los estudiantes correspondientes a las carreras de Publicidad, Relaciones Públicas y Licenciatura en Negocios, Diseño y Comunicación del turno tarde de la Facultad de Diseño y Comunicación de la Universidad de Palermo. Esta materia se ubica en el segundo año en las carreras de Publicidad y Relaciones Públicas permitiendo a los estudiantes formarse en el conocimiento y manejo de herramientas metodológicas propias de los ambientes académicos y necesarios para la elaboración de trabajos de investigación, de trabajos de profundización teórica y para la comprensión y crítica de publicaciones científicas. En la Licenciatura en Negocios, Diseño y Comunicación la materia se encuentra ubicada en el segundo semestre acompañando a los estudiantes en el afianzamiento de los conocimientos metodológicos adquiridos en las respectivas carreras de grado, como así también los orienta y ejercita en el diseño, proyecto y concreción de un trabajo de investigación. En ambos casos también les provee los elementos necesarios para comprender, utilizar y evaluar trabajos de investigación, resultados de estudios de relevamiento y manejo de datos cuantitativos como cualitativos propios del ámbito laboral y valioso para el desempeño profesional.

Esta materia forma parte del grupo Ensayos Contemporáneos cuyos objetivos se vinculan a la realización de documentos escritos de acuerdo a las normas académicas y del desarrollo de las habilidades necesarias para la reflexión sobre temáticas vinculadas al acontecer actual, propias de la producción teórica de un ámbito universitario que se encuentra en continuo proceso de elaboración y producción de cuestionamientos y posiciones que cuestionan y se plantean acerca de los fenómenos que marcan y caracterizan el momento actual. En este sentido se busca no sólo que los estudiantes puedan realizar un trabajo de investigación sino que puedan concretar sus resultados en un documento académico que permita vincular los resultados hallados con la elaboración conceptual correspondiente. Se busca ejercitarlos en la elaboración y producción de documentos escritos de acuerdo a las normas de presentación vigentes en la Universidad de Palermo y que responden a las normas de presentación general de un documento académico y de nivel científico.

Esta investigación se enmarca en el área de comunicación en relación a los diversos dispositivos que la posibilitan y en el estudio de algunos de los efectos que resultan de su utilización por parte de los sujetos, en su faceta de usuarios ya sea activos como pasivos. A esta altura los estudiantes se encuentran iniciando o manejando diversas teorías de la comunicación como también, a través de materias específicas de la currícula de cada carrera, utilizando diversos modelos conceptuales, que necesitan ser ubicados dentro del paradigma teórico que les da sentido, para a la vez, poder ser puestos en cuestionamiento y permitir el pasaje hacia un proceso de comprensión, a través de la desnaturalización de las certezas y la realidad aceptada facilitando cierta incomodidad y desapego del fenómeno para que pueda ser puesto en evidencia y desde ese lugar poner en suspensión la propia postura para permitir ampliar la perspectiva y formular nuevas posibilidades. Dado que la población estudiantil es mayormente joven, se ha buscado una temática, que a través de su vigencia, les permita a estos estudiantes ejercitarse en el estudio de fenómenos propios de la actualidad, permitiendo posicionar a la tarea de adquisición de conocimientos y el desarrollo de la investigación en un ámbito apropiado para generar curiosidad e interés ingredientes favorecedores para el desarrollo de nuevas áreas de crecimiento (con miras a un mejor rendimiento académico y proveyendo herramientas que faciliten un mejor posicionamiento a futuro como profesional).

La presente propuesta tiene repercusiones en otras áreas de la currícula en relación a dos cuestiones: por un lado provee los elementos, normas y encuadre necesarios para elaborar un trabajo con el adecuado rigor metodológico .En este sentido la cursada, brinda herramientas para comprender y analizar el valor de una 
producción académica en su relación con la producción científica y en los diferentes caracteres que la misma sustenta: consistencia lógica, provisoriedad, objetividad, sujeción a prueba, rigor metódico y sistemático, capacidad para describir, explicar y predecir, a través del ejercicio del análisis y la crítica rigurosa. El uso de un lenguaje pertinente, claro y preciso es necesario para lograr una comunicación que restrinja la ambigüedad y favorezca la neutralidad valorativa. Por otro lado, proporciona un ambiente facilitador para adquirir la experiencia de realizar un trabajo de investigación, que desde su inicio, diseño y proyecto permita transformar ese aprendizaje, en un encuentro con la tarea y el conocimiento que se produce en el espacio áulico y se proyecta a los otros espacios de elaboración y producción: encuentros extrauniversitarios, trabajos de biblioteca, consulta a otros profesionales, etc. Esta experiencia es a su vez un eslabón importante, como antecedente tanto para los trabajos particulares de las otras materias que impliquen la elaboración de documentos escritos como para los trabajos de final de grado y de licenciatura.

El presente trabajo está centrado en las denominadas Tecnologías de la información y la comunicación (TIC) usadas por jóvenes universitarios entre dieciocho y veintiocho años. Estos dispositivos y los usos derivados de los mismos son parte del modo de habitar la actualidad. En este sentido, como todo fenómeno novedoso, es inquietante y genera corrientes que los glorifican como otras que los ubican como generadores de procesos productores de malestar y dañinos para la vida en sociedad. Considerando la institución universitaria como un ámbito adecuado para la observación, el estudio y el análisis de fenómenos contemporáneos, se propone analizar algunos de los modos en que estos dispositivos afectan los procesos de subjetivación en especial los referidos a la construcción, sostenimiento y finalización de vínculos significativos. Desde un punto de vista macrosocial, estas tecnologías también son fruto no sólo de la investigación sino también de una organización económica particular orientada hacia el consumo, modalidad que afecta los modos de pertenencia social, como así también nuevas formas de exclusión que se manifiestan de diversas maneras de acuerdo a las distintas clases sociales y pertenencia a diferentes grupos etarios, de género, nacionalidad, entre otros. Los trabajos realizados por los estudiantes están focalizados en dos de esas tecnologías: el celular e internet y en las modalidades que adquieren estos dispositivos en relación a algunos de los vínculos significativos divididos en dos tipos: los que refieren a sujetos y los que refieren a instituciones. Dentro del primer tipo los trabajos están centrados en los vínculos con: familias de origen, pares (amistad), parejas (novios, parejas estables, amantes). Dentro del segundo tipo se ubican los vínculos que se dan en los ámbitos institucionales más comunes en este rango etario: instituciones educativas e instituciones laborales.

\section{Presentación del Proyecto}

\section{Fundamentación}

Uno de los aspectos que sustenta mi práctica académica se refiere a la posibilidad de comprender, cuestionar y ampliar la propia perspectiva del mundo a través de la producción académica. En este sentido la materia Metodología de Investigación se posiciona en un lugar privilegiada ya que busca, a través de sus diversos contenidos, agitar las opiniones, sacudir las posturas adquiridas y generar un espacio de encuentro que facilite la discusión y el intercambio de las ideas, junto con la adquisición de nuevos conocimientos.

En este sentido todas las actividades están orientadas a lograr que los estudiantes puedan construir y generar un lugar, que en su sentido antropológico, implica un territorio poblado de marcas identificatorias, históricas y culturales. El aula se constituye en una encrucijada que marca el cruce con lo propio y lo ajeno de la institución 
universitaria, de la materia, de los otros compañeros de cursada y del docente. En este sentido habilita un encuentro con conocimientos y herramientas que generalmente se encuentran poco apreciadas por los estudiantes debido a cierta resistencia inicial para el encuentro con el esfuerzo y el tiempo que implica la labor con conceptualizaciones teóricas en su función de modelos/intentos de explicación de los diversos fenómenos que nos afectan y que construyen eso que llamamos realidad. También es el encuentro/desencuentro con un universo discursivo y simbólico propio de la materia. A ello se suma las situaciones generadas por las diferentes pertinencias de origen, usos del vocabulario y las modalidades del dictado de esta materia.

Paralelamente el aula también se constituye en un espacio, noción abstracta que se relaciona con las nociones de extensión territorial, salto en el tiempo y lapso temporal. Como extensión demarcable el aula es un lugar concreto que a su vez se constituye en un espacio para el aprendizaje. Cada uno de los partícipes tiende a acomodarse en el mismo y a transformar ese espacio en un lugar que facilite el desarrollo de las diversas habilidades y actividades necesarias para cumplimentar la cursada. En el sentido temporal el encuentro áulico semanal implica también un lapso, una ausencia necesaria para el trabajo y la elaboración de las diversas actividades que implica esta instancia del aprendizaje. En este sentido, el espacio como corte actualiza la idea de proceso, noción que incluye tiempo en su doble significado tanto referido a la continuidad como a la discontinuidad, necesarios para el trabajo de comprensión y también para la elaboración de trabajos de investigación. Todas las actividades de la cursada están organizadas y planificadas de una manera gradual, en función de la realización del trabajo de investigación, teniendo en cuenta los siguientes: Objetivos Generales:

1. Iniciar y familiarizar a los estudiantes con el trabajo de investigación.

2. Afianzar el hábito del pensamiento y la discusión teórica.

3. Reconocer y articular los aportes teóricos de los autores con el trabajo de investigación Objetivos Específicos:

4. Identificar y conocer los conceptos básicos de metodología de investigación.

5. Elaborar y desarrollar un proyecto de investigación.

6. Conocer y aplicar instrumentos para la recolección de datos.

7. Manejar e interpretar datos de tipo cualitativo.

8. Reconocer e identificar abordajes teóricos para la comprensión de la realidad. El logro de estos objetivos se encuentra relacionado con la posibilidad también de desarrollar la idea de encuentro con el aprendizaje, con lo diferente y ajeno que esta materia proporciona. Incluye también la noción de oportunidad, circunstancia que transforma lo semejante e igual, en único y creativo. En función de estos aspectos, el tránsito por la cursada se convierte en un desafío para transformar, generar y posibilitar que los estudiantes se constituyan en sujetos habilitados para transformar la curiosidad en una herramienta para la creación y la producción.

También refiere a la valorización del espacio áulico como un lugar que promueve el conocimiento a partir, no sólo de las acciones del docente (explicación y desarrollo de conceptos, acompañamiento en las diferentes actividades, evaluación de los estudiantes) sino también de las acciones que se generan a partir de los 
estudiantes, entre ellos y con la docente. Durante la cursada se promueven diversos modos de participación: planteamiento de preguntas y cuestiones, debates, exposición de temas, intercambios de propuestas de trabajo, elaboración y exposición de trabajos domiciliarios, ejercicios de búsqueda de material y elaboración de diferentes instancias vinculadas a la producción de trabajos de investigación. Estas actividades buscan promover el desarrollo de habilidades vinculadas a la comunicación: escritura, exposición y retórica, cualidades que conforman parte de los procesos de la producción científica, ya que la divulgación facilita la transformación de un conjunto de ideas en aquello denominado conocimiento científico.

Por ello, el diseño, proyecto y realización de un trabajo completo de investigación en cursada, cuya aprobación es requisito indispensable (junto con otros instrumentos) para obtener la habilitación para rendir el final. Esta condición quiere decir que los alumnos deben completar todas las instancias establecidas para ello, lo cual incluye una corrección final una vez entregado el mismo.

La realización de este trabajo permite a los estudiantes cumplimentar dos tipos de objetivos generales: - Los referidos a la incorporación de conocimientos y habilidades de la Metodología de Investigación: aplicando los conocimientos explicados y desarrollados durante la cursada, reconociendo el aporte de la investigación a las distintas disciplinas, adquiriendo (a través del ejercicio y del trabajo) las nociones fundamentales sobre qué es y cómo se hace una investigación con rigor científico, produciendo un documento académico o informe final (que incluye el desarrollo de habilidades de escritura y de exposición oral).

- Los referidos a la conceptualización, incorporación y desarrollo de conceptualizaciones teóricas vinculadas a los fenómenos de lo actual. En este sentido, las Tecnologías de la Información y la Comunicación (TIC) propuestas; el uso del celular y de internet y los dispositivos que las portan, como diría Perriault (1989) "las máquinas de comunicar" abren preguntas sobre los modos que los sujetos habitamos lo actual, visibilizado a través de las prácticas que se generan a partir del uso de ciertos dispositivos mostrando las diferentes modalidades que adquieren problemas de larga data en la humanidad, a la vez que enfatiza los diferentes grados de frustración y malestar que generan. El paradigma que sustenta las conceptualizaciones actuales se ha transformado. La complejidad plantea fenómenos y sujetos posicionados en un tiempo que ha transformado las nociones de presente y de futuro. Marcados por la incertidumbre (fundamentalmente tras la caída acerca de las certezas sobre el futuro de la humanidad), complementariedad ((una realidad puede ser conceptualizada de maneras diferentes sin que ello genere la primacía o la exclusión de la otra posición) y el contextualismo (la realidad varía según la perspectiva desde la cual la observamos). Es importante señalar que también hablamos de un sujeto comprometido y responsable por la realidad en la que está inmerso, donde los diferentes fenómenos tienen una comprensión holística, y cuyos productos científicos son parciales no por incompletos sino porque sus explicaciones (mas allá del cumplimiento de las normas científicas) se encuentran limitadas por el valor y la capacidad de explicar sectores de la realidad.

\section{Tipología}

Este proyecto áulico muestra el desarrollo y concreción de trabajos de investigación en cursada vinculados a problemáticas actuales. Brinda la posibilidad de estudiar, dentro del marco del diseño cualitativo, los usos que los jóvenes universitarios hacen de algunas de las nuevas tecnologías de la comunicación. Para este primer cuatrimestre de 2011 la temática elegida es: "Jóvenes y tecnologías de la información y la comunicación. Usos y consumos: el amor, la amistad y el trabajo en jóvenes universitarios entre 18 y 28 años. Estos usos estarán 
centrados (como se señaló con anterioridad) en relación a vínculos significativos con familias de origen (y/o), con pares (y/o), pareja (y/o), educativos (y/o) laborales.

\section{Necesidad que cubre}

La cumplimentación de este proyecto permite una modalidad superadora de algunas de las dificultades que aparecen cuando se realizan trabajos de investigación. Puede señalarse que los aspectos que generalmente producen mayores dificultades están centrados en: el recorte temático, elaboración de hipótesis, operacionalización de las variables, análisis y procesamiento del trabajo de campo, elaboración del informe final, finalización y entrega satisfactoria del trabajo completo de acuerdo a los tiempos previstos. Este proyecto acompaña, a través de la cursada, todos los momentos críticos de la realización de este tipo de trabajos, facilitando la adquisición de hábitos y modalidades metodológicas, entendidas como las estrategias que permiten cumplir con los objetivos e incrementar los conocimientos.

Cada una de estas situaciones tiene en la planificación académica un lugar para su cumplimentación, que incluye actividades en clase y extraclase que se plasman en los diferentes avances que el trabajo tiene estipulado y en la entrega de diferentes trabajos prácticos, que luego conformarán el corpus del portfolio de cursada.

Ha resultado una herramienta muy valiosa el seguimiento clase a clase de los diferentes avances, generado, no sólo, por la presentación del material establecido sino por la posibilidad de su discusión, reelaboración y reescritura en el tiempo de la clase. La clase se convierte en un laboratorio de producción, que no está centrado en forma exclusiva en la exposición docente. Se convierte en un espacio de trabajo, en el cual, el docente se ubica más en la tarea de tutorear la actividad y favorecer que los estudiantes desarrollen y ejerciten las habilidades propias de un investigador universitario.

También la observación de los trabajos entre los compañeros de cursada, el intercambio sobre avances y fuentes, y la exposición grupal de distintos aspectos de la investigación ha mejorado notablemente las producciones.

No es ajeno al ámbito universitario, la incorporación de la tecnología, ya que muchos estudiantes trabajan directamente en sus laptops, ello permite rastrear e investigar fuentes, conectarse con otros ámbitos de investigación y favorecer la curiosidad y profundización temática. El manejo de las mismas y sus avatares, también facilita la interacción entre los estudiantes aspecto que mejora la integración, favoreciendo la escucha y el trabajo con lo diferente y a veces extraño, que el compañero porta. El manejo de la tecnología se hace presente, también, durante las presentaciones que tienen carácter de evaluación parcial. La utilización de las mismas es un interesante apoyo para las exposiciones orales, trabajándose la cantidad de información que deben contener para no generar contaminación visual, que encandile y desvalorice la exposición oral.

Los tiempos y su cumplimiento, vehiculizan las diferentes fases que implica todo trabajo de investigación, convirtiéndose en un "tempo", que marca el ritmo de las diferentes actividades. La integración de estos tiempos de producción y su concreción, a través de los trabajos prácticos de cursada ha permitido que los estudiantes adquieran, en forma acompañada, el rigor y la organización necesarias (en el sentido metodológico) para concretar un trabajo de investigación. 
Un elemento importante, en términos de productividad, es el clima de trabajo que comienza a instalarse en el ámbito de la clase. Clima que incluye una modalidad de trabajo orientada al logro de una tarea, que implica la espera, el proceso, trabajar junto a otros compañeros en temas y actividades diversas, tolerando que la docente esté por tiempos con cada grupo y que la actividad se encuentre de alguna manera descentrada y focalizada en las acciones que cada grupo pueda realizar en el espacio semanal asignado a esta materia.

\section{Organización de la cátedra}

Todas las actividades del cuatrimestre están orientadas al logro de los objetivos propuestos. La estructura y planificación de cada una de las clases, adquiere modalidades específicas de acuerdo a los logros necesarios para cumplimentar al final del cuatrimestre el trabajo de investigación.

Como estructura general, puede señalarse que las actividades están concentradas en dos módulos temporales. El primero centrado en la adquisición y comprensión de los conceptos teóricos necesarios para comprender el valor de la investigación científica y su ubicación en el contexto cultural, en cuanto a la producción de nuevos sentidos. Un segundo módulo que focaliza en el ejercicio de la tarea del investigador y en las diversas acciones que su ejercicio requiere. En cuanto a las actividades de clase, éstas están planificadas en actividades teóricas y prácticas. Durante el desarrollo del primer módulo los tiempos asignados a la explicación y desarrollo de conceptos son mayores que los de la ejercitación práctica. En el segundo momento las explicaciones teóricas van perdiendo gradualmente la centralidad temporal, adquiriendo mayor relevancia el trabajo en clase y la orientación tutorial se convierte en el eje de la tarea docente.

Las actividades y tareas de los estudiantes están organizadas en actividades que van desde la labor individual hasta la grupal.

\section{Docente}

Este proyecto está liderado por la Profesora Débora Belmes, que tiene a su cargo esta cátedra. Las responsabilidades de la docente están centradas: en las propias de la actividad pedagógica y en el logro de los objetivos establecidos para el cuatrimestre, cuya concreción puede evaluarse a través de la presentación de los trabajos de investigación realizados por los estudiantes.

\section{Estudiantes}

Los estudiantes son informados desde el inicio de la cursada de las modalidades de la misma, ello incluye el uso de la documentación escrita consistente en: la planificación académica, las guías para los trabajos prácticos, el programa analítico y las guías para el primer parcial y el trabajo práctico final (ver anexo).

Los estudiantes que participaron en este trabajo son:

a) Que se encuentran cursando carreras de grado: Andrade Almachi, Christian (Ecuador, 24 años, Lic. en Publicidad); Asturias Guzman, Nicole (Guatemala, 20 años, Lic. en Publicidad); Barreira, María Noelia (Argentina, 23 años, Lic. en Relaciones Públicas); Bianchi, Laura Angela (Argentina, 22 años, Lic. en Relaciones Públicas); Burtin, María Paula (Argentina, 24 años, Lic. en Relaciones Públicas); Cabrera Franchi, Stefanía Carolina (Venezuela, 21 años, Lic. en Publicidad); Carro, Vanina Soledad (Argentina, 21 años, Lic, en 
Relaciones Públicas); Casas, Rodrigo (Argentina, 19 años, Lic. en Publicidad); Castillo Gomez, Miriam Cecilia (Uruguay, 33 años, Lic. en Publicidad); Cerra, Melina Beatriz (Argentina, 25 años, Lic. en Relaciones Públicas); Haisner, Fernanda Giselle (Argentina, 21 años, Lic. en Relaciones Pú- blicas); Herrera Cordova, Claudia Carolina (Peru, 20 años, Lic. en Relaciones Públicas); Lopez Veloso, Tomás Agustin (Argentina, 21 años, Lic. en Publicidad); Mahanna, Marwa (Canadá, 23 años, Lic. en Relaciones Públicas); Muller, Marina Ayelen (Argentina, 20 años, Lic. en Relaciones Públicas); Rivero, Fernando Andrés (Argentina, 19 años, Lic. en Publicidad); Rivero, Axel (Argentina, 29 años, Lic. en Relaciones Públicas); Sagray, Daniela (Argentina, 20 años, Lic. en Relaciones Públicas); Spagnuolo, Micaela Belén (Argentina, 25 años, Lic. en Relaciones Públicas); Velasco Velez, Leonela Yajseel (Ecuador, 18 años, Lic. en Publicidad).

b) Cursantes de la Licenciatura en Negocios, Diseño y Comunicación: Falcon Moscoso, Maribel Roxana (Perú, 30 años); Rivas Hermoso, Miriam Gisela (Venezuela, 29 años); Rodriguez Wilches, Germán (Colombia, 27 años); Scalbi, Ornela (Argentina, 22 años).

Equipos de Trabajo Los estudiantes se organizaron de diversas maneras (desde individual, pasando por pares, tríos y grupales) para cumplimentar el trabajo de investigación. La conformación de los equipos de trabajo fue libre y se consideró fija a partir de la cuarta clase. Todos los grupos tenían las mismas consignas generales, es decir las vinculadas a la realización de un trabajo de investigación y particulares, las vinculadas al recorte temático elegido (aspectos detallados en el ítem referido a la planificación académica). De ello resultaron los siguientes temas que fueron trabajados por los equipos, cuyos títulos e integrantes aparecen a continuación: Una nueva prueba de amor: Rivas Miriam, Scalbi, Ornela Facebook y la ruptura de parejas: Falcon, Maribel; Rodriguez Wilches, German; Bianchi Laura.

Los jóvenes y las tecnologías de la información y la comunicación: Herrera, Carolina; Cabrera, Stefanía; Asturias, Nicole.

Usos del celular y jóvenes: Andrade Christian.

Los jóvenes: técnica de estudio clásica o informática: Castillo, Cecilia, Velasco Velez, Leonela. El teléfono tiene la palabra: Mahanna, Marwa; Cerra, Melina.

Redes y medios de comercialización: Haisner, Fernanda; Carro, Vanina; Muller, Marina. Jóvenes y consumos culturales: el uso de smartphones y las redes sociales: Burtin; María Paula; Barrera Noelia; Rivero, Fernando; Sagray, Daniela; Spagnuolo, Micaela.

Redes y adicción: Rivero, Axel; Casas, Rodrigo; Lopez Veloso, Tomás.

\section{Corpus}

Poder investigar implica adquirir herramientas y habilidades específicas. Por ello se parte de un encuadre general para poder lograr una aproximación al foco de la materia: investigar. La elección de un tema implica centrar esta perspectiva, y organizar el aprendizaje en función de este objetivo.

Todas las acciones de la planificación están pensadas desde esta perspectiva sin olvidar la articulación teóricopráctico, necesaria para aprobar la materia. 
Durante el primer cuatrimestre de 2011, el desarrollo de los módulos tanto teórico como práctico se desplegó a lo largo de quince clases, que adquirieron diversas modalidades que se detallan a continuación.

El primer módulo se desarrolla durante las tres primeras clases. La impronta teórica es fuerte, buscando que los estudiantes adquieran ciertos posicionamientos teóricos vinculados a: ciencia, investigación en sus diferentes acepciones como pura, orientada, técnicas y como desarrollo tecnológico, métodos científicos, epistemologías y paradigmas cientí- ficos. Estos conceptos se vehiculizan a través de la exposición oral de la docente (explicación, desarrollo y ejemplificación de los diferentes desarrollos conceptuales), la realización de actividades de ejercitación en clase cuyo fin es la apropiación de los conceptos, su debate y crítica. Este módulo temático requiere la realización de dos trabajos prácticos: el primero titulado "Ciencia y Vida cotidiana" busca promover la curiosidad y la observación de la presencia de la ciencia en el diario acontecer, desde su perspectiva teórica como a través de sus productos, descubrimientos y contradicciones. El ejercicio implica el trabajo con publicaciones periodísticas, su análisis para establecer relaciones y su justificación con los diferentes conceptos teóricos expuestos. En este primer ejercicio se busca no sólo que los estudiantes puedan aplicar los conceptos al análisis del material por ellos elegido, sino que también comienzan a prestar atención sobre los fenómenos que acontecen y son publicados, buscando generar curiosidad, trabajando con los propios valores y prejuicios. En relación al trabajo de investigación se busca favorecer el uso de las diferentes concepciones teóricas al modo de herramientas, que como tales permiten cierta apropiación de la realidad, su uso y transformación. El segundo trabajo práctico denominado "Los métodos en la ciencia" posee también diferentes finalidades. La finalidad específica se vincula al afianzamiento de las concepciones teórico-metodológicas trabajadas en clase. La finalidad general refiere al manejo de diferentes fuentes teóricas y la elaboración de fichas de lectura. Esta actividad les permite a los alumnos apropiarse de un instrumento metodológico que facilita el trabajo con múltiples fuentes. Por otro lado, la confección de este trabajo los enfrenta a la tarea de producir un documento que refiere al tratamiento que distintos autores producen acerca de ciertos conceptos que son caracterizados desde posiciones diferentes. En este sentido el trabajo comienza a enfrentarlos con la diversidad científica y con el ejercicio del pensamiento, la abstracción de las ideas fundamentales y la ausencia de una verdad absoluta y determinista.

En la cuarta clase comienzan a desarrollarse los temas específicos y necesarios para el desarrollo de una actividad de investigación, cada encuentro puntualiza y desarrolla los aspectos que permiten cumplimentar la cursada, realizar un trabajo y facilitar el manejo de los nuevos conceptos del universo simbólico propio de la metodología de la investigación. La cuarta clase tiene como ejes temáticos las nociones de proceso de investigación, diseño y proyecto. Ello implica trabajar con los sentidos atribuidos a cada una de estas nociones, el valor y la diferenciación que estos conceptos adquieren en relación a la labor del investigador en su relación con distintas instancias de evaluación. Se caracterizan los distintos niveles de investigación y se describen y explican las características de trabajos cualitativos y cuantitativos. También se desarrolla las diferentes fases que todo proceso de investigación contiene: planteamiento y formulación, diseño del universo, diseño del objeto, recolección de datos, análisis y procesamiento, elaboración del informe y exposición. Junto con las fases se comienzan a enunciar las cuatro instancias de validación conceptual que conforman un proceso de investigación. En esta clase comienza a trabajarse con la temática propuesta por la cátedra. Las actividades de los estudiantes están orientadas a elaborar un primer recorte temático y un plan de trabajo. Para estas actividades podrán organizarse de manera individual o grupal. Esta primera organización grupal implica la incorporación de una modalidad de trabajo en clase orientada al debate, la confrontación y la producción de 
ideas como fruto del trabajo grupal. Para ello deberán cumplir con diversas tareas: elaborar un primer punteo de temas posibles, en un segundo momento la problematización de los mismos y la elaboración de preguntas que intentan definir y acotar las líneas temáticas, distinguir la relación de grupos de preguntas con temas diversos y la distinción de problemas y subproblemas.

Este ejercicio también les permite enfrentarse y trabajar con la propia posición ideológica, que muchas veces aparece en forma de prejuicios y el debate con las diferentes posiciones que los otros miembros del grupo detentan. Cada producción grupal o individual es luego volcada al grupo total, actividad que favorece la escucha hacia los pares, permite apreciar la diversidad de abordajes que un tema puede suscitar y comienza a instalar una dinámica que antecede y prepara para las exposiciones orales. En esta misma clase se comenzará a delinear el proyecto de investigación en función de la planificación de las diversas actividades que deben cumplimentar a través de los trabajos prácticos que tienen fechas de entrega previamente establecidas. La labor iniciada en clase debe ser luego transformada en el trabajo práctico número tres. En este trabajo deben presentar el cronograma de actividades que den cuentan de los diversos avances de la investigación, determinando los tiempos estimados para cada una de las labores, las fechas de finalización estimadas para cada una de ellas, las etapas del proceso que deben ser cumplimentadas y detallando los distintos documentos que deberán presentar de acuerdo a los requisitos de la cursada. Este trabajo deberá incluir la definición del primer recorte temático, que incluirá la elaboración de la situación problemática elegida, su definición y una primera distinción entre problemas y subproblemas. La quinta clase focaliza el trabajo con la noción de problema comenzada a trabajar en la clase anterior e introduce las nociones de hipótesis, objetivos y marco teórico. Nociones necesarias para ahondar en el planteamiento y formulación de un proceso de investigación, que como tal, busca dar respuestas a una problemática planteada. Estas nociones son conceptualizadas en una relación dinámica, donde la afectación e influencia, es, en esta instancia del trabajo, absolutamente interdependiente. Si bien, el trabajo con estas nociones requiere definiciones precisas, el trabajo conceptual se orienta en un primer momento a la explicación y desarrollo de sus distintas definiciones y características, y en un segundo momento también se busca detallar la función e importancia de estos conceptos en el desarrollo de la labor investigativa. El trabajo con las hipótesis implica comenzar a elaborar las nociones de variable, valor de la variable y definición operacional de las variables. Conceptos complejos que no se agotan en esta aproximación sino que requiere sucesivos abordajes vehiculizados a través del desarrollo y avance en el trabajo de investigación.

La comprensión de estas nociones comienza a desarrollarse a partir del trabajo con las mismas, en su aplicación al tema elegido de investigación. Para ello, se utilizan como base los trabajos comenzados la clase anterior, y completados en el lapso interclase. Como este material requiere una primera corrección, los estudiantes se organizan en sus grupos de trabajo para comenzar a aplicar los conceptos desarrollados al material aportado. La docente circula por los diferentes grupos observando la planificación presentada y evaluando, en una primera mirada, la pertinencia temática, la justificación presentada. En un segundo momento, se evaluará también los aportes que este tipo de problemática posibilita. Mientras la docente rota por los grupos, los estudiantes comienzan a elaborar los primeros objetivos e hipótesis que serán también supervisados por la docente. Puede señalarse que la docente puede circular por un mismo grupo varias veces en la misma clase. Comenzar a trabajar en un recorte temático específico, posibilita el encuentro con diferentes abordajes disciplinares, que estarán conectados con el primer momento del marco teórico, que consiste en la búsqueda de fuentes que dan cuenta del denominado estado de la cuestión (entendiendo por tal, la descripción de las principales líneas 
teóricas que explican esa problemática, cuáles son los principales autores, y cuáles han sido las líneas de investigación desarrolladas).

El trabajo práctico número cuatro titulado "El proceso de investigación: el marco teórico y las hipótesis" permite plasmar en un documento las actividades que requiere la búsqueda y elaboración del marco teórico vinculado al recorte temático elegido. Esta actividad implica la visita a la biblioteca de la facultad y el rastreo de otros materiales teóricos como fuentes conceptuales. Esta actividad deberá configurar una lista tentativa de autores, resultado del rastreo bibliográfico. Esta lista luego será trabajada por cada grupo en función de la pertinencia e importancia que cada elaboración puede aportar a la comprensión de los fenómenos estudiados. Este trabajo práctico también debe incluir la elaboración y selección de objetivos (en función de las actividades que el investigador desarrollará a lo largo del trabajo) y le enunciación de por lo menos dos hipótesis.

Como puede observarse, los trabajos prácticos son iniciados en clase y luego son modificados, completados y transformados en documento, en el tiempo que media entre una clase y la siguiente. Lapso que facilita el procesamiento de la información y permite el decantamiento de los conceptos y desarrollos más importantes.

La sexta clase es el momento del primer parcial. Este consiste en un trabajo de aplicación de conceptos teóricos al análisis de una película seleccionada dentro de una lista propuesta por la docente. Este trabajo que se plasma en un documento escrito (cuyas consignas se encuentran detalladas en una guía denominada "Guía para el primer parcial") debe ser presentado en forma oral, frente a todos los compañeros. El tiempo para cada presentación es de diez minutos y pueden utilizar el cañón para ilustrar la exposición. Esta evaluación permite una primera aproximación a la presentación de un trabajo tanto en forma escrita como oral. Incluye la aplicación de conceptos al análisis de un material, en este caso el discurso cinematográfico, que como medio vehiculiza esta ejercitación, facilitando el manejo conceptual, desarrollando y ampliando las habilidades necesarias para el análisis y procesamiento de diferentes fenómenos, como una aproximación a su comprensión. También transforma, a los conceptos propios de una jerga particular, en herramientas para la apropiación y transformación de un discurso particular, en un medio para la comprensión de las teorías como herramientas para la comprensión del mundo.

La séptima clase se centra en los conceptos de universo y muestra. Implica trabajar también con las nociones de muestreo aleatorio y no aleatorio. Estos conceptos, vinculados a la tercera fase del proceso de investigación focalizan en los sujetos de la investigación, en sus protagonistas, en quienes encarnan la problemática propuesta. Este trabajo permite seguir profundizando las nociones de unidad de análisis, variables y valores de la variable, Este trabajo a su vez, modifica las hipótesis propuestas, generando nuevas definiciones de las mismas y la corrección también de los objetivos. Estos aspectos requieren bastante ejercitación y reelaboración. La asistencia docente se convierte en una labor de ida y vuelta, donde a través de los distintos señalamientos, se generan nuevas enunciaciones, que requieren nuevas correcciones.

Estas correcciones, a su vez, transforman el recorte, favoreciendo ciertas miradas disciplinares, que reorientan la búsqueda bibliográfica y comienza a delinear el marco teórico propiamente dicho de cada trabajo de investigación.

En la clase número nueve se explican los instrumentos que permiten la obtención y construcción de aquello que se denomina dato. Esta clase está relacionada con la cuarta fase del proceso de investigación denominada: 
diseño del instrumento. Se describen instrumentos tanto para trabajos cualitativos como cuantitativos.

Se profundiza y detallan las características de los instrumentos vinculados a los trabajos de tipo cualitativo: la observación, la entrevista, los grupos de opinión, las historias de vida, el análisis documental. La entrevista semiestructurada, es detallada en forma particular, ya que es el instrumento elegido para este trabajo de investigación.

Durante la clase se realiza una ejercitación (rollplaying) vinculada a la implementación de las entrevistas semiestructuradas y también las dificultades que genera.

Luego se trabaja por grupos, las diferentes líneas temáticas, que deberían recorrer las entrevistas, en función de la hipótesis elegida y del trabajo con sus variables. A su vez, se chequea el estado de las actividades planificadas, el armado del marco teórico y las dificultades con la operacionalización de las variables.

El trabajo práctico correspondiente, denominado "El proceso de investigación, el trabajo de campo" requiere dos actividades: la elaboración de una ficha de lectura (herramienta ya trabajada) sobre la entrevista semiestructurada y la realización de dos entrevistas, cuyo registro escrito funcionará a modo de prueba piloto de este instrumento. Desde el nivel conceptual, todo instrumento debe ser chequeado, a través de pruebas piloto, para ajustar su pertinencia, mejorar su eficacia. Desde un nivel pragmático, acerca a los estudiantes al ejercicio de realizar entrevistas y los desafíos que este instrumento plantea.

La clase número diez comienza con el trabajo aportado por las experiencias en la realización de las entrevistas. Un representante de cada grupo lee a todos los compañeros del curso, la entrevista realizada. Los demás compañeros deben tratar de señalar cuáles son los temas principales y aportar críticas y sugerencias para lograr una mayor eficacia y la obtención de más elementos para ser transformados en datos. La escucha de los pares, favorece la interacción y el aprendizaje, mejorando la calidad de las entrevistas con miras a un mejor producto de investigación.

En esta misma clase se comienzan a delinear los elementos que incluye la elaboración de un informe final de investigación desde las partes que lo conforman hasta los elementos que contiene. Se comienza a trabajar en el proceso de escritura que en paralelo incluye, el trabajo con el análisis y el procesamiento de los datos. En este sentido, la elaboración del informe los confronta con el procesamiento del material, por lo cual se trabajan ambas líneas a la vez.

En clase se comienzan a esbozar la elaboración de un índice definitivo para el trabajo final, con la consecuente determinación de partes. El comenzar a trabajar en el índice, favorece la visualización del trabajo, y jerarquiza el lugar que el análisis y procesamiento de los datos, tienen en un trabajo de investigación. También se chequean los tiempos y las actividades, en función de la planificación, señalando los aspectos a revisar y las falencias en cuanto a la elaboración del trabajo.

La dinámica de la clase, continúa adjudicando mayor tiempo al trabajo grupal y a la rotación de la docente, facilitando las correcciones en los distintos trabajos.

En la clase once, se continua explicando y desarrollando los aspectos conceptuales vinculados a la elaboración de un informe de investigación. También se trabaja con las normas de presentación académica (normas APA) y 
la conformación de la bibliografía. Se ejemplifica las citas, las notas al pie, la utilización de páginas electrónicas, etc. Se trabaja sobre el índice definitivo del trabajo y se comienza a delinear el borrador final, que será presentado en la clase siguiente. Este borrador deber incluir contenidos y esbozo básico de la introducción, el cuerpo del trabajo y las conclusiones.

La clase doce, está focalizada en el trabajo con los borradores del trabajo su revisión y consulta. Constituye la última corrección posible antes de la entrega de la versión definitiva. Se trabaja intensamente sobre los contenidos de la guía del trabajo práctico final (ver anexo) y los ítems que debe incluir el informe escrito. También se repasan las características y tiempos para la exposición oral.

La clase trece es el tiempo para la presentación de los trabajos de investigación frente a todo el curso. Cada grupo presenta sus trabajos, responde a las preguntas y comentarios de sus compañeros. La docente escucha todas las presentaciones y toma nota, grupo por grupo de los modos en que la exposición oral toma cuerpo. Para ello se consideran los siguientes ítems: presentación de los integrantes de cada grupo, presentación del tema, organización y administración del tiempo, claridad expositiva, uso de lenguaje acorde y técnico, desarrollo de lo presentación (inicio, desarrollo y conclusiones). La presentación de estos trabajos y su exposición oral llevan nota, y ello equivale al segundo parcial. Esta clase cierra con una evaluación general de lo acontecido en la misma, por parte de los estudiantes y de la docente.

En la clase catorce la docente hace una devolución general de los trabajos presentados señalando las características comunes, los logros y los déficits. Se entrega a cada grupo el informe escrito con las correcciones correspondientes. Se otorga un período de tiempo para la lectura de las correcciones y luego grupo por grupo se trabajan las mismas con la docente. Es importante este momento ya que la versión corregida del trabajo debe ser presentada a la clase siguiente para obtener la habilitación para el final. El tiempo que media entre la lectura y la corrección y devolución por parte de la docente a cada grupo, es también aprovechado para comentar y observar los informes escritos por los otros compañeros de cursada.

La clase quince consiste en la entrega de los trabajos revisados (las dos versiones del cuerpo $\mathrm{B}$, la corregida y la definitiva) y en el cierre y evaluación de la cursada.

\section{Cronograma}

Los tiempos para cada una de las actividades se encuentran articulados con los estimados en la planificación académica. En el siguiente cronograma se detallan los principales momentos vinculados al trabajo de investigación.

Clase 1: Se informa la temática a investigar. Se enuncia el TP 1(ver anexo).

Clase 2: Entrega del TP1 Clase 3: Se trabaja sobre los métodos en ciencia. Se enuncia el TP2 (ver anexo).

Clase 4: Se comienza a trabajar con el proyecto de investigación. Trabajo en pequeños grupos. Se comienza a delinear el TP 3 (ver anexo).

Clase 5: Entrega del TP3. Se trabajan sus contenidos para poder enunciar las primeras hipótesis y objetivos. Se comienza a delinear el marco teórico tentativo. Se enuncia el TP 4 (ver anexo). 
Clase 6: primer parcial. Exposición oral de trabajos de análisis realizados como parcial domiciliario (ver punto corpus $\mathrm{C}$ y anexo, guía para el primer parcial).

Clase 7: Entrega del TP 4. Se continúa con el trabajo de investigación. Se trabaja sobre las unidades de análisis que conforman la muestra. Se ajustan los elementos presentados en los trabajos prácticos anteriores.

Clase 8: Se trabaja sobre la entrevista semiestructurada. Se realizan rollplaying de entrevistas. Se enuncia el TP5 (ver anexo).

Clase 9: Entrega del TP 5. Se leen las entrevistas correspondientes a los trabajos prácticos. Se marcan los aciertos y se sugieren mejoras y correcciones en función de cada uno de los temas. Se comienza a trabajar en las modalidades de análisis y procesamiento de los datos.

Clase 10: Se trabaja sobre el índice del trabajo y el contenido de los capítulos. Son trabajos que se realizan grupo por grupo. La docente tiene una función tutorial.

Clase 11: Se continúa con el trabajo por grupo y comienza a delinearse el borrador del trabajo. Se enuncia el TP 6 (ver anexo).

Clase 12: Se presenta el TP 6. Se trabaja en forma grupal y se ajustan y revisan el contenido de los borradores presentados.

Clase 13: Presentación de los trabajos de investigación. Exposición oral de cada uno de los grupos (ver corpus C y anexo, guía para la entrega del trabajo práctico final).

Clase 14: Entrega de los trabajos corregidos. La docente realiza una devolución grupo por grupo donde se revisan y ajustan los elementos señalados para lograr un trabajo adecuado.

Clase 15: Entrega final de los trabajos de investigación. Los estudiantes deben entregar los trabajos de acuerdo a las normas establecidas y con una copia digital (PDF).

\section{Evaluación}

Los mecanismos de evaluación formal de la cursada están centrados en los trabajos prácticos y en las dos presentaciones con carácter de parcial. Los criterios establecidos para su evaluación implican por un lado, cumplir con los requisitos y consignas establecidas en cada una de las guías correspondientes y por otro lado, la capacidad para no sólo cumplir la consigna sino establecer relaciones y análisis vinculados a un buen manejo teórico junto con una adecuada redacción. Cada trabajo práctico lleva nota (de uno a diez), y además deben ser completados e incluso vueltos a confeccionar dentro de la cursada para cumplimentar con las consignas establecidas. Los trabajos prácticos que están directamente relacionados con el trabajo de investigación (a partir del tercer trabajo) se comienzan a confeccionar en clase, facilitando el intercambio con los compañeros y la docente. Ello incluye en algunas oportunidades, la lectura de lo elaborado que permite la colaboración de los compañeros tanto para profundizar las líneas de trabajo, como para orientar y ampliar las perspectivas. Esos trabajos son completados en el lapso entre clase y entregados en la siguiente. La docente los corrige, pone nota y señala sugerencias y orientaciones al trabajo presentado. La observación, escucha y participación en 
presentaciones áulicas se convierte también en un motor que si bien, no constituye una evaluación formal, actúa como si lo fuera, movilizando a los estudiantes a mejorar la producción de sus trabajos.

El primer parcial es un trabajo de elaboración domiciliaria (ver Corpus, sexta clase). Consiste en la aplicación de conceptos teóricos al análisis de una película (dentro de una lista propuesta por la docente) que deben plasmarse en un documento escrito. Este análisis debe ser presentado en la sexta clase frente a todos los compañeros. El tiempo para cada presentación es de diez minutos y pueden ilustrarla con el uso del cañón. Las consignas y criterios para su evaluación se encuentran detallados en la Guía para el primer parcial. Estos criterios incluyen la presentación en tiempo y forma del trabajo y la exposición oral; dedicación y preparación de acuerdo a las consignas y finalmente se evalúa la calidad discursiva (uso y aplicación de los conceptos teóricos) tanto del Trabajo Escrito como en la Presentación Oral. En la Presentación Oral, se evalúa la organización de la misma, el tiempo general y el atribuido a cada expositor, la claridad de los conceptos y la profundidad del análisis. La nota de este parcial es una nota promedio entre la presentación oral y el informe escrito.

El segundo parcial constituye la presentación de los trabajos de investigación (ver Corpus, clase trece). Las presentaciones son frente a todos los compañeros de cursada. Los elementos que se consideran relevantes son: presentación del grupo, sus integrantes y del tema a desarrollar, organización y administración del tiempo, claridad expositiva, coherencia, uso de lenguaje acorde al ámbito universitario, desarrollo de la presentación de acuerdo a las consignas (introducción, desarrollo y conclusiones). Los criterios establecidos en la Guía correspondiente refieren que la evaluación considerará: la presentación en tiempo y forma, dedicación y preparación del informe tanto en su elaboración como en su análisis, calidad tanto del trabajo como de su presentación, actitud y argumentación en la defensa del Trabajo, respeto de las normas de presentación de los trabajos en Diseño y Comunicación y el cumplimiento de las consignas especificadas en la guía.

Los trabajos escritos y su presentación oral llevan nota que se promedia constituyendo un valor de uno a diez.

Una vez finalizadas todas las exposiciones correspondientes a cada parcial, se realiza una evaluación grupal de la experiencia. En la misma se intercambian impresiones, opiniones e ideas fruto de la participación en esta actividad áulica. Los estudiantes señalan las ventajas de incorporar y comprender conceptos mediante este tipo de ejercitación y también manifiestan cierta sorpresa al percibir que el esfuerzo que implica investigar agrega cierto gusto y placer a la tarea de descubrir, utilizar y comprender ciertos fenómenos vinculados a la propia realidad.

\section{Informe / Ensayo}

La época actual puede ser definida de diversas maneras pero es inevitable considerar que las imágenes, los sonidos y las pantallas se constituyen en elementos propios del escenario cotidiano. Estos elementos podrían ser agrupados dentro del concepto de dispositivo. Agamben (Agamben, 2005) define al dispositivo como "cualquier cosa que tenga de algún modo la capacidad de capturar, orientar, determinar, interceptar, modelar, controlar y asegurar los gestos, las conductas, las opiniones y los discursos de los seres vivientes". Señala que a la proliferación de dispositivos equivale una proliferación de modos de subjetivación. En este sentido puede señalarse que en este escenario se desarrolla aquello que denominamos vida cotidiana. Ésta puede ser entendida como un entramado de historia pasada y presente que se encuentra en proceso de transformación generando actividades y quehaceres para dar sentido a lo nuevo. Las nuevas herramientas comunicacionales 
muestran y magnifican los circuitos de reconocimiento y afecto, las pertenencia grupales, la importancia de la inclusión y de la exclusión, como también la transformación de las nociones de lo público y de lo privado a partir de la expansión de los diversos dispositivos.

Un punto central en este análisis es la existencia de la red, que desde su polisemia, transmite la idea de contención, de medio, de intercambio y de ardid.

La red permite delinear un mundo de vínculos, que contiene y sostiene, frente al desamparo, la caída de los referentes sociales y la fragmentación subjetiva. También es un medio que soporta los cambios geográficos, la velocidad de los procesos, la simultaneidad de los fenómenos y la incertidumbre del mundo contemporáneo. Es posible señalar que si bien la mayoría de los jóvenes tiene celular, por ejemplo, los inmigrantes/migrantes, si no lo tienen, se convierte en uno de los primeros objetos a adquirir. Los trabajos realizados por los estudiantes permiten señalar ciertas inferencias. Por ejemplo que si bien "chatear" y pertenecer a una red social, son espacios propios del mundo "joven", la palabra escuchada (sus tonos y modalidades) adquieren relevancia frente a situaciones en las que necesitan mayor contención, y/o también cercanía. En el caso de los migrantes se observa que la voz aparece (por los menos en los primeros tiempos) como un bálsamo frente a un escenario en el que no se terminan de arraigar y en el que lo nuevo no terminar de armarse como propio. La palabra escuchada también facilita una comunicación más directa, ya que sus modalidades permiten dotar de sentido al contenido de lo dicho. El uso del celular marca una diferencia interesante en cuanto a lo público y lo privado. El mail y/o pertenecer a una red social es un dato que puede darse a cualquiera, pero el teléfono se encuentra ligado a vínculos significativos tanto desde el punto de vista afectivo (familiares, amigos, parejas) como desde el punto de vista laboral. El uso del celular también ha mostrado cierta invasión de lo privado en el mundo de lo público. Permite localizar al interlocutor, "hola ¿cómo va?, ¿dónde estás?” dando lugar a esta categorización actual de "medios locativos" (que puede estar asociada a cierta idea de control y mapeo cotidiano) como también ubicar quien llama (diferentes sonidos). El celular también invade el espacio público con sus sonidos y conversaciones, desconociendo a esos otros que pueblan ese lugar y generando situaciones de imposición sonora y temática como incomodidad frente a los contenidos de sus mensajes.

Conectar, comunicar, chatear/charlar, realizar una operación comercial, pueden configurarse como un encuentro con el otro, donde la geografía tradicional es olvidada, y un escenario virtual propicia nuevos sentidos. Estos medios ya no implican un punto fijo en la geografía, sino que justamente dan cuenta de cierta movilidad asociada a la velocidad de los desplazamientos pero que no se constituye en un impedimento temporal para lograr una conexión. Ello nos permite preguntarnos si estamos ante una nueva forma de pensar al tiempo y al espacio, categorías que históricamente han permitido organizar la vida en sociedad y que sentidos va adquiriendo la idea de conexión.

La red facilita un tiempo fluido, que permite la deriva contenida. Es omnipresente y paradojalmente intermitente. Los jóvenes usan las redes, no sólo para estar conectados (que hay un otro posible) sino que estas permiten comentar, mostrar, y armar diversas redes que contienen y retienen en la posible deriva y posiblemente frenen cierto sentimiento de soledad. En este sentido sería válido preguntar la contracara de estos fenómenos. ¿Tanta conexión, no estará dando cuenta de la dificultad para profundizar y sostener vínculos? ¿Tendrá que ver con el desamparo frente a tanta abundancia de objetos y cierta fragmentación subjetiva? Muchos jóvenes manifestaron preocupación por las horas dedicadas a "estar en la red" y en las dificultades sociales que ello provocaba: aislamiento, confusión, deterioro de los vínculos cara a cara, Por otro lado, las redes e internet, se constituyen 
en elementos propios del hacer laboral y educativo. Su presencia en los distintos ámbitos es incuestionable y favorece no sólo la búsqueda de información sino también el desarrollo de diversas actividades, la apertura a nuevos mercados laborales como también la adquisición e intercambio de información y conocimientos.

La presencia virtual del otro permite que nos preguntemos si afecta y/o renueva el sentido a la ausencia, al silencio. Los contactos a través de las redes, favorecen la posibilidad también del engaño, la mentira y la exposición (buscada y no buscada). El malentendido genera dificultades con los seres queridos. Pero aquí habría que preguntarse si ello es generado por las limitaciones de este dispositivo, o es que ¿es propio de la comunicación y requiere de otras especificidades para que pueda desanudarse? El grupo comprendido entre los 18 y 24 años pareciera estar más dispuesto a participar e incluir aspectos de la intimidad sin mediar mayores reparos. Los jóvenes comprendidos entre los 25 y 28 años dedican menos tiempo a la deriva virtual, además de privilegiar y seleccionar que datos publicar.

A su vez, la velocidad y la simultaneidad, ¿afectan los tiempos y los procesos? ¿Borrar a alguien de la lista, es hacer desaparecer los afectos que lo acompañaron? Muchos de los jóvenes entrevistados comentaron que la red es un medio que permite dar a conocer novedades: estas pueden incluir presentar un nuevo partenaire/novio/pareja como también anunciar la finalización de la misma. En este sentido su anuncio no vela ni excluye los tiempos vinculados a su procesamiento. Muchos refirieron la polifonía de los contactos frente a estos cambios de "status". ¿Estaremos frente a diferentes formas de procesar la finalización de vínculos significativos? El tiempo virtual puede ser compartido con otros y con otras tecnologías que no entran en competencia sino que se complementan. Conectar, agruparse configuran la antesala del encuentro con el otro, encuentro que pone en juego lo propio y lo ajeno, lo conocido y lo nuevo. En este sentido las nuevas tecnologías plantearían nuevos modos de construir sostener distancias, espacios, lo común y lo compartido. La red en este sentido es polifónica, si prevalencia de lo vertical sobre lo horizontal. La posibilidad de formar parte de diversos grupos no requiere mayores trámites como también la posibilidad de desligarse de los mismos.

Todos los trabajos aportaron la idea, que si bien estos dispositivos y los modos de conexión con el otro tienen mucho de virtual, ello no reemplaza el encuentro cara a cara. Más bien, implica la antesala, frente a lo desconocido, y el encuentro virtual, frente a la imposibilidad geográfica. Podría señalarse que ello está relacionado con algo de lo paradojal de estos dispositivos: acercan y alejan, juntan y aíslan, encuentran y desencuentran. Estos dispositivos muestran, exageran y subrayan las complejidades de iniciar, sostener y finalizar vínculos significativos. También nos fuerzan a armar y repensar los modos de estar en vínculos con otros, en tiempos caracterizados por este modo de habitar lo actual.

\section{Conclusiones}

Este trabajo permite apreciar que se puede aprender a investigar en cursada a través de la realización de un trabajo de investigación. Los estudiantes pueden por un lado, incorporar las nociones propias de la materia, a través del ejercicio de las tareas propias y a la vez, aprender y desarrollar conceptualizaciones con rigor teórico que les permiten analizar fenómenos del mundo contemporáneo. En este sentido puede señalarse, que la elección del tema ha sido de relevancia, ya que permite a los jóvenes amplificar la curiosidad y ejercer el trabajo de diferenciar la simple opinión de la crítica fundada en la conceptualización teórica. Por otro lado, la organización de la cursada y de sus diversas actividades facilita el logro de los objetivos. También puede 
señalarse que el espació áulico ha sido fundamental como espacio de encuentro que organiza, ejercita y facilita el desarrollo de las diversas tareas.

En términos generales puede señalarse que el proceso de investigación puede cumplimentarse si las actividades de la cursada están organizadas para ese fin. En este sentido la planificación se constituye en una herramienta fundamental, no sólo en el puro sentido pedagógico, sino también en el sentido práctico, pues es allí donde están condensados los temas y actividades fundamentales propuestas para ese período.

Investigar es una tarea compleja, comprenderlo y atravesarlo es propio de un ambiente académico. Por ello el aula ha constituido en esta experiencia un lugar fundamental: favorece los intercambios, permite el seguimiento, facilita la consulta (con la docente y entre pares), estimula la producción. En este sentido, la incorporación del espacio del aula, como un tiempo de trabajo (que implica elaboración, consulta, búsqueda, escucha, intercambio, corrección, entre otros) ha facilitado el tránsito por los momentos críticos del proceso de investigación: recorte temático, elaboración de hipótesis, análisis del material de campo y elaboración del informe. El clima de trabajo (áulico) merece una mención. Es un clima que se va construyendo clase a clase y que implica la incorporación y el procesamiento de elementos y situaciones que vinculan las tareas de investigar con el tiempo y la responsabilidad propia de cada grupo por hallar un camino que permita transformar la curiosidad y el cuestionamiento a la realidad, en una serie de propuestas que validen ciertos hallazgos.

Dentro de las dificultades puede señalarse que la primera refiere a la incorporación del ritmo necesario para cumplimentar el trabajo en cursada. En este sentido puede señalarse que los trabajos prácticos, fuerzan a sostenerlo, pero al principio ello genera cierto desasosiego manifestado en dificultades para profundizar las temáticas, recortar los temas y elaborar las hipótesis. También apropiarse del trabajo áulico, e incorporar el pasaje de un docente de tipo expositor a uno que trabaja más desde la actividad de tutor, también requiere cierto tiempo y genera resistencias, grupos que consultan con más frecuencia que otros, dificultades para administrar el tiempo para cumplimentar los trabajos, aprovechamiento del encuentro justamente donde la actividad convocante es el trabajo de investigación. Otra de las dificultades refiere a vencer el prejuicio sobre "lo aburrido y denso" que es investigar. Ello requiere de tiempo, búsqueda y motivación a través del recorte temático y sostenimiento a través de los cuestionamientos que los mismos estudiantes van generando sobre la temática elegida. Dentro de los hallazgos puede señalarse que el uso de las computadoras personales ha facilitado el trabajo grupal, fundamentalmente en términos de elaboración y corrección. También ha permitido a los estudiantes observar y revisar los distintos avances, promover la ampliación y comparación de las nociones conceptuales e intercambiar fuentes con otros compañeros de cursada.

Este modelo de trabajo muestra que esta experiencia puede replicarse para temáticas específicas, de corto desarrollo temporal a través de una clara articulación de las actividades en tiempos de entrega y logros específicos para cada momento. También es importante señalar que el acompañamiento docente clase a clase y la consulta facilitan la tarea creando un clima de trabajo beneficioso para el mismo. Al finalizar los estudiantes se encuentran, sorprendidos de sus propias producciones y de los hallazgos que sus trabajos arrojan. En este sentido, la propuesta de trabajo vinculada a ampliar, cuestionar la propia perspectiva del mundo se encuentra ampliamente alcanzada.

Este proyecto también permite mostrar que los estudiantes son sujetos habilitados a transformar la curiosidad en una herramienta para la producción y la creación, y que el ambiente académicos es un buen catalizador para 
ello.

Bibliografía

Agamben, G. (2007). Infancia e Historia. Buenos Aires: Adriana Hidalgo Editora.

Agamben, G. (2005). ¿Qué es un dispositivo? Buenos Aires: Conferencia en la UNLP, 12/10/05.

http//www.trelew.gov.ar/web/files/LEF/SEM03-Agamben-QueesunDispositivo.pdf Ander Egg E. (1983). Técnicas de investigación social. Buenos Aires: Editorial El Cid.

Auge, M. (1992). Los “no lugares”. Barcelona: Gedisa (1994).

Bauman, Z. (2007). Vida de consumo. Buenos Aires: Fondo de cultura económica.

Berardi, F. (2007). Generación post-alfa: patologías e imaginarios en el semiocapitalismo. Buenos Aires: Tinta Limón.

Diaz E. (1997). Metodología de las Ciencias Sociales. Buenos Aires: Biblos.

(1999). Posmodernidad. Cap. 1. Buenos Aires: Editorial Biblos.

Giddens, A. (1993). Consecuencias de la modernidad. Madrid: Alianza Universidad.

Ivoskus D. (2008). Vivir conectados. Buenos Aires: Grupo Editorial Norma.

Morin, E. (1994). Introducción al pensamiento complejo. Barcelona: Gedisa.

Perriault, J. (1991). Las máquinas de comunicar y su utilización lógica. Barcelona: Gedisa.

Rheingold, H. (2002). Multitudes inteligentes. Barcelona: Gedisa.

Sabino C (1994). El Proceso de Investigación. Buenos Aires: Editorial Lumen/Humanitas.

Taylor y Bogdan (1992). Introducción a los métodos cualitativos de investigación. Buenos Aires: Paidos.

Wainerman, C. y Sautu, R. (2001). La trastienda de la investigación. Buenos Aires: Ediciones Lumiere S.A.

Winocur, R (2009). Robinson Crusoe ya tiene celular. Mexico: Siglo XXI; U A Metropolitana, unidad Iztapalapa.

1.2. Nuevas herramientas de la comunicación. Un estudio acerca del amor, la amistad, la educación y el trabajo en jóvenes universitarios fue publicado de la página 156 a página176 en Cuadernos del Centro de Estudios de Diseño y Comunicación № 63 